\title{
Retos de futuro en el tratamiento del juego patológico
}

\author{
Enrioue Echeburúa
}

Facultad de Psicología. Universidad del País Vasco.

Enviar correspondencia a:

Enrique Echeburúa. Facultad de Psicología. Universidad del País Vasco. Avda. de Tolosa, 70. 20018 San Sebastián. E-mail: eecheburua@ss.ehu.es

\section{RESUMEN}

En este artículo se señalan los principales avances y los retos de futuro en el tratamiento de la ludopatía. Los instrumentos de evaluación del juego patológico actualmente disponibles, específicos (autoinformes, como el SOGS, o entrevistas clínicas, como el NODS) o más generales, como el ASI referido a la ludopatía, son aún muy limitados. Los estudios referidos a la terapia del juego patológico prueban que este cuadro clínico responde bien a los tratamientos ofertados, con unas tasas de éxito entre el $50 \%$ y el $80 \%$ de los casos tratados en un seguimiento a largo plazo. En concreto, la terapia de elección parece ser el control de estímulos y la exposición en vivo con prevención de respuesta, seguida por una intervención cognitivo-conductual en prevención de recaídas. La terapia psicofarmacológica puede ser complementaria cuando los pacientes tienen un estado de ánimo deprimido o un elevado nivel de impulsividad. El juego controlado puede ser una alternativa terapéutica para los jugadores jóvenes o que no presentan aún una dependencia severa. Se requiere aún más información sobre las mujeres jugadoras y sobre los programas terapéuticos más específicos para ellas. Se comentan las implicaciones de esta revisión para la práctica clínica y para las investigaciones futuras en este campo, así como las limitaciones actualmente existentes.

Palabras clave: Juego patológico. Instrumentos de evaluación. Tratamientos psicológicos. Tratamientos farmacológicos. Prevención de recaídas.

\section{ABSTRACT}

This paper deals with the new developments in the treatment for pathological gambling, as well as with the challenges for further research. Assessment tools currently available, such as self-reports (SOGS or ASI) or clinical interviews (NODS) have many limitations. Reviewed studies about treatment of pathological gambling show that this disorder improves significantly with proposed therapies, with a success rate ranging from $50 \%$ to $80 \%$ of treated patients in a long-term follow-up. However, the choice treatment appears to be stimulus control and in vivo exposure with response prevention, followed by a cognitive-behavioral intervention in relapse prevention. Psychopharmacological therapy may have incremental benefit when patients have comorbid depression or high impulsivity. Responsible gambling may be a therapeutic option for young gamblers or people without a severe dependence. Further information is required about women gamblers and about the most convenient programs for them. The relevance of this review for clinical practice and future research in this field, as well as the unsolved problems, are commented upon.

Key words: Pathological gambling. Assessment measures. Psychological treatment. Pharmacological treatment. Relapse prevention. a ludopatía es una forma de adicción sin droga. Las adicciones no se limitan exclusivamente a las conductas problemáticas generadas por algunas sustancias químicas, como los opiáceos, los estimulantes, los ansiolíticos, la nicotina o el alcohol. Lo cierto es que de conductas normales -incluso saludables-, como jugar, comer o utilizar el ordenador, se pueden hacer usos anormales en función de la intensidad, de la frecuencia o de la cantidad de dinero invertida $y$, en último término, en función del grado de interferencia negativa en las relaciones familiares, sociales y laborales de las personas implicadas (Echeburúa y Corral, 1994).

Las adicciones sin drogas funcionan, en unos casos, como conductas sobreaprendidas que traen consigo consecuencias negativas y se adquieren a fuerza de repetir comportamientos que en un principio resultan agradables; en otros, como estrategias de afrontamiento inadecuadas para hacer frente a los 
problemas personales (por ejemplo, acudir al bingo para hacer frente a la ansiedad o a la tristeza).

La presencia incontrolada de máquinas tragaperras en bares y lugares de ocio, junto con la oferta abundante de bingos, casinos y de los juegos más tradicionales (quinielas, loterías, cupones, carreras de caballos, etcétera), ha hecho aumentar considerablemente la ludopatía. La indefensión ante esta avalancha de juegos con apuestas afecta especialmente a los adolescentes y a las personas más vulnerables psicológicamente.

En España, la tasa de prevalencia de la ludopatía oscila entre el $2 \%$ y el $3 \%$ de la población adulta (Becoña, 1999; Irurita, 1996; Tejeiro, 1996). El trastorno es mucho más frecuente en hombres que en mujeres, pero éstas son mucho más reacias a buscar ayuda terapéutica por la censura social existente. A diferencia de otras conductas adictivas, el juego patológico se distribuye por todas las clases sociales y por todas las edades. No obstante, la edad de acceso al juego ha descendido en los últimos años. De hecho, cada vez son más los adolescentes que buscan tratamiento por problemas de juego (Báez y Echeburúa, 1995; Becoña, 2001).

Los ludópatas, al menos en España, muestran una dependencia fundamentalmente a las máquinas tragaperras, ya sea sólo a éstas o en combinación con otros juegos. La dependencia en exclusiva a otros juegos de azar es mucho menor (Báez, Echeburúa y Fernández-Montalvo, 1994). Este hecho no es fruto de la casualidad, sino que obedece a una serie de aspectos psicológicos implicados en el funcionamiento de este tipo de máquinas (Echeburúa, 1992): su amplia difusión; el importe bajo de las apuestas con posibilidad de ganancias proporcionalmente cuantiosas; la brevedad del plazo transcurrido entre la apuesta y el resultado; la manipulación personal de la máquina (que genera una cierta ilusión de control); y las luces intermitentes de colores, que, junto con la música y el tintineo estrepitoso de las monedas, suscitan una tensión emocional y una gran activación psicofisiológica.

Desde una perspectiva psicopatológica, la depresión y el abuso de alcohol están asociados con frecuencia al juego patológico (Báez et al., 1994; McCormick, Russo, Ramírez y Taber, 1984). De hecho, la tasa de prevalencia del consumo abusivo de alcohol u otras drogas entre los ludópatas oscila alrededor del 15\%-20\% (Báez et al., 1994; Lesieur y Heineman, 1988; Rodríguez-Martos, 1989).

Los instrumentos de evaluación del juego patológico actualmente disponibles son muy limitados. De hecho, la investigación sobre la efectividad de los enfoques terapéuticos no ha estado basada en criterios uniformes, por lo que resulta difícil comparar los distintos estudios (Toneatto y Ladouceur, 2003). En el ámbito clínico se cuenta con instrumentos de screening, como el Cuestionario Breve de Juego Patológico (CBJP) (Fernández-Montalvo, Echeburúa y Báez, 1995), con pruebas más propiamente diagnósticas, como el Cuestionario de Juego de Soath Oaks (SOGS) (Lesieur y Blume, 1987; validación española de Echeburúa, Báez, Fernández-Montalvo y Páez, 1994) o, más recientemente, con entrevistas clínicas basadas en los criterios del DSM-IV, como la NORC DSM Screen for Gambling Problems (NODS) (Gerstein, Hoffman y Larison, 1999). Por otra parte, un instrumento de interés en el ámbito de las adicciones, como el Índice de Severidad de la Adicción (ASI) (McLellan, Luborsky, Woody y O'Brien, 1980), ha sido adaptado recientemente al ámbito del juego patológico, analiza el nivel global de funcionamiento del paciente en la vida diaria y cuenta con buenas propiedades psicométricas (Petry, 2003).

Los estudios referidos a la terapia del juego patológico prueban que este cuadro clínico responde bien a los tratamientos ofertados. Desde una perspectiva general, se han obtenido unas tasas de éxito superiores al $50 \%$ de los casos tratados con un control mínimo de seguimiento de 6 meses (Echeburúa, Fernández-Montalvo y Báez, 1999a; Echeburúa y Fernández-Montalvo, 2003; Toneatto y Ladouceur, 2003). Además, esta tasa de éxito puede aumentar incluso hasta el $80 \%$ cuando se aplican técnicas conductuales -control de estímulos y exposición en vivo con prevención de respuesta, principalmente- seguidas de una intervención cognitivo-conductual en prevención de recaídas (cfr. Echeburúa, Fernández-Montalvo y Báez, 1999b, 2000). En la actualidad este tipo de técnicas constituyen, sin duda, el tratamiento de elección de la ludopatía.

Aun siendo un tema polémico, el empleo de técnicas cognitivas, defendido con ardor por diferentes autores (cfr. Toneatto y Ladouceur, 2003), no parece ser tan necesario para conseguir la abstinencia del juego. Independientemente del número elevado de distorsiones cognitivas que presentan los jugadores patológicos (cfr. Fernández-Montalvo, Báez y Echeburúa, 1996), la eficacia de los tratamientos a largo plazo no aumenta cuando se incluyen este tipo de intervenciones cognitivas (cfr. Echeburúa, Báez y Fernández-Montalvo, 1996).

Si bien el tratamiento psicológico es prioritario en el ámbito de la ludopatía, queda pendiente, desde una perspectiva psicofarmacológica, el estudio de las situaciones concretas en las que el empleo de medicación (por ejemplo, la paroxetina u otro antidepresivo IRSS), de forma combinada con la terapia psicológica, potencia la eficacia terapéutica -jugadores con ánimo deprimido o con un nivel elevado de impulsividad, por ejemplo- (Ibáñez y Saiz, 2000). Sin embargo, los resultados obtenidos hasta la fecha son todavía incon- 
cluyentes (Grant y Kim, 2002; Grant, Kim y Potenza, 2003).

Hay muchas cuestiones que no están todavía suficientemente resueltas. Por ejemplo, el estudio de la ludopatía en función del sexo se ha hecho de una forma muy incompleta. En general, los hombres tienden a jugar más por excitación y para ganar dinero; las mujeres, para hacer frente a problemas personales (soledad, depresión, etcétera) y familiares. Pero estas diferencias no se han tenido en cuenta a la hora de diseñar tratamientos específicos. De hecho, son muy pocas las mujeres que han formado parte de las investigaciones clínicas publicadas hasta la fecha. También resultaría de interés diseñar tratamientos concretos para tipos de juegos específicos (Raylu y Oei, 2002).

Asimismo un reto de futuro es diseñar estrategias motivacionales para el tratamiento con el objetivo de atraer a la terapia a personas que niegan o minimizan su dependencia del juego (Hodgings, Currie y ElGuebaly, 2001; Hodgings, Currie, El-Guebaly y Peden, 2004), así como hacer un estudio específico de los abandonos del tratamiento (Ladouceur, Grosselin, Laberge y Blaszczynski, 2001). Más allá de la prevención de recaídas, la retención de los pacientes durante el tratamiento constituye una prioridad. La tasa de abandonos puede oscilar en torno al 20\% (Báez, Echeburúa y Fernández-Montalvo, 1995; Echeburúa, FernándezMontalvo y Báez, 2001). Por ello, la entrevista motivacional y las estrategias adecuadas a las fases del cambio del paciente, según el modelo de Prochaska y DiClemente (1982), constituyen un camino prometedor. En este sentido ha habido un primer estudio exploratorio aplicando este enfoque para mejorar la retención de los pacientes en el tratamiento cognitivo-conductual de la ludopatía (Milton, Crino, Hunt y Prosser, 2002). Los resultados son, sin embargo, todavía provisionales.

El juego patológico no constituye un constructo unitario. Por ello, establecer tipologías de jugadores patológicos es una tarea muy sugerente porque va a posibilitar establecer estrategias terapéuticas diferenciadas en función de las mismas. Ha habido algunos intentos de clasificar a los ludópatas en puros, emocionalmente vulnerables e impulsivos (Blaszczynski y Nower, 2002) o en jugadores buscadores de activación, jugadores buscadores de dinero y jugadores de alivio o de escape (Prieto, 2003) o, por último, en función de la gravedad de la psicopatología asociada (González, Aymami, Jiménez, Doménech, Granero y Lourido-Ferreira, 2003), pero se requiere aún un mayor apoyo empírico a estas clasificaciones.

Desde otra perspectiva, resulta de interés el tratamiento de los trastornos duales. En las investigaciones controladas sobre la terapia del juego patológico se utilizan, por razones metodológicas, jugadores puros, sin otro trastorno psicopatológico concomitante. No obstante, la experiencia clínica demuestra la existencia, en numerosas ocasiones, de comorbilidad e incluso de un diagnóstico dual en el ámbito de la ludopatía (alcoholismo, esquizofrenia y deficiencia mental, principalmente, en el eje I; trastorno antisocial y borderline de la personalidad, en el eje II) (Blaszczynski y Steel, 1998; Fernández-Montalvo y Echeburúa, 2001, 2004). En estos casos, las técnicas terapéuticas propuestas no se muestran tan útiles como en los jugadores puros. Se requiere más investigación a este respecto.

Asimismo hay un interés actual por analizar la viabilidad del juego controlado como objetivo terapéutico en el tratamiento de la ludopatía (Ladouceur, Sylvain, Boutin, Lachance, Doucet y Leblond, 2003). Los resultados obtenidos no son concluyentes. Al margen de las propuestas demasiado optimistas de algunos autores, quizá sea éste un objetivo válido para ciertos pacientes (los jugadores problemáticos), pero no para otros (los ludópatas propiamente dichos).

En otras palabras, la adaptación de los objetivos clínicos (abstinencia total o juego controlado) y de los programas de tratamiento a las necesidades individuales, así como el papel terapéutico mismo de los manuales de autoayuda (por ejemplo, Blaszczynski, 1998, Milton, 2001 o Hodgins, 2002, en el ámbito anglosajón, y Fernández-Montalvo y Echeburúa, 1997, en el ámbito hispanohablante), constituyen una cuestión no resuelta que requiere una detallada investigación.

Por último, una cuestión de gran interés es la referida a las diferencias individuales. ¿Necesitan todos los jugadores patológicos tratamiento para controlar su hábito? Aunque prácticamente no hay investigaciones controladas sobre la evolución espontánea de los pacientes no tratados (cfr. Hodgins y El-Guebaly, 2000; Nathan, 2003), algunos, como ocurre también en el caso de ciertos bebedores excesivos y de ciertos fumadores, pueden dejar de jugar por sus propios medios; otros, por el contrario, no responden ni al mejor de los tratamientos disponibles. En el estudio de Echeburúa, Báez y Fernández-Montalvo (1994), por ejemplo, el $25 \%$ de los pacientes pertenecientes al grupo de control habían dejado de jugar a pesar de no haber recibido ninguna intervención terapéutica durante un período de 6 meses.

\section{REFERENCIAS}

Báez, C. y Echeburúa, E. (1995). El control de estímulos y la exposición con prevención de respuesta como tratamiento psicológico de la ludopatía en un adolescente.

Análisis y Modificación de Conducta, 21, 125-145. 
Báez, C., Echeburúa, E. y Fernández-Montalvo, J. (1994). Características demográficas, de personalidad y psicopatológicas de los jugadores patológicos de máquinas tragaperras en tratamiento: un estudio descriptivo. Clínica y Salud, 5, 289-305.

Báez, C., Echeburúa, E. y Fernández-Montalvo, J. (1995). Variables predictoras de abandonos y recaídas en el tratamiento del juego patológico. Análisis y Modificación de Conducta, 21, 5-22.

Becoña, E. (1999). Epidemiología del juego patológico en España. Anuario de Psicología, 30, 7-19.

Becoña, E. (2001). Juego problema y juego de riesgo en adolescentes. Adicciones, 13, 279-288.

Blaszczynski, A.P. (1998). Overcoming compulsive gambling. A self-help guide using cognitive behavioral techniques. London. Robinson.

Blaszczynski, A.P. y Steel, Z. (1998). Personality disorders among pathological gamblers. Journal of Gambling Studies, 14, 51-71.

Blaszczynski, A.P. y Nower, L. (2002). A pathways model of problem and pathological gambling. Addiction, 97, 487-499.

Echeburúa, E. (1992). Psicopatología, variables de personalidad y vulnerabilidad psicológica al juego patológico. Psicothema, 4, 7-20.

Echeburúa, E., Báez, C. y Fernández-Montalvo, J. (1994). Efectividad diferencial de diversas modalidades terapéuticas en el tratamiento psicológico del juego patológico. Análisis y Modificación de Conducta, 20, 617-643.

Echeburúa, E., Báez, C., Fernández-Montalvo, J. y Páez, D. (1994). El Cuestionario de Juego de Soath Oaks (SOGS): validación española. Análisis y Modificación de Conducta, 20, 769-791.

Echeburúa, E., Báez, C. y Fernández-Montalvo, J. (1996). Comparative effectiveness of three therapeutic modalities in the psychological treatment of pathological gambling: long-term outcome. Behavioural and Cognitive Psychotherapy, 24, 51-72.

Echeburúa, E. y Corral, P. (1994). Adicciones psicológicas: más allá de la metáfora. Clínica y Salud, 5, 251-258.

Echeburúa, E., Fernández-Montalvo, J. y Báez, C. (1999a). Avances en el tratamiento del juego patológico. Adicciones, 11, 349-361.

Echeburúa, E., Fernández-Montalvo, J. y Báez, C. (1999b). Prevención de recaídas en la terapia del juego patológico: eficacia diferencial de tratamientos. Análisis y Modificación de Conducta, 25, 375-403.

Echeburúa, E., Fernández-Montalvo, J. y Báez, C. (2000). Relapse prevention in the treatment of slot-machine pathological gambling: long-term outcome. Behavior Therapy, 31, 351-364.

Echeburúa, E., Fernández-Montalvo, J. y Báez, C. (2001). Predictors of therapeutic failure in slot-machine pathological gamblers following behavioural treatment. Behavioural and Cognitive Psychotherapy, 29, 379383.

Echeburúa, E. y Fernández-Montalvo, J. (2003). Nuevas perspectivas en el tratamiento del juego patológico. Revista Thomson de Psicología, 1, 139-155.

Fernández-Montalvo, J., Báez, C. y Echeburúa, E. (1996). Distorsiones cognitivas de los jugadores patológicos de máquinas tragaperras en tratamiento: un análisis descriptivo. Cuadernos de Medicina Psicosomática y Psiquiatría de Enlace, 37, 13-23.

Fernández-Montalvo, J. y Echeburúa, E. (1997). Manual práctico del juego patológico. Ayuda para el paciente y guía para el terapeuta. Madrid. Pirámide.

Fernández-Montalvo, J., Echeburúa, E. y Báez, C. (1995). El Cuestionario Breve de Juego Patológico (CBJP): un nuevo instrumento de screening. Análisis y Modificación de Conducta, 21, 151-179.

Fernández-Montalvo, J. y Echeburúa, E. (2001). Trastornos de personalidad y juego patológico: una revisión crítica. Psicología Conductual, 9, 527-539.

Fernández-Montalvo, J. y Echeburúa, E. (2004). Pathological gambling and personality disorders: An exploratory study with the IPDE. Journal of Personality Disorders, 18, 500-505.

Gerstein, D., Hoffman, J., Larison, C. et al. (1999). Gambling Impact and Behavior Study. Chicago. National Gambling Impact Study Commission.

González, A., Aymami, M.N., Jiménez, S., Doménech, J.M., Granero, R. y Lourido-Ferreira, M.R. (2003). Assessment of pathological gamblers who use slot machines. Psychological Reports, 93, 707-716.

Grant, J.E. y Kim, S.W. (2002). Pharmacotherapy of pathological gambling. Psychiatric Annals, 32, 186191.

Grant, J.E., Kim, S.W. y Potenza, M.N. (2003). Advances in the pharmacological treatment of pathological gambling. Journal of Gambling Studies, 19, 85-109.

Hodgins, D.C. (2002). Becoming a winner. Defeating problem gambling. Calgary, Canadá.

Hodgins, D.C., Currie, S.R. y El-Guebaly, N. (2001). Motivational enhancement and self-help treatments for problem gambling. Journal of Consulting and Clinical Psychology, 69, 50-57.

Hodgins, D.C., Currie, S.R. , El-Guebaly, N. y Peden, N. (2004). Brief motivational treatment for problem gambling: 24 month follow-up. Psychology of Addictive Behaviors, 18, 293-296.

Hodgins, D.C. y El-Guebaly, N. (2000). Natural and treatment-assisted recovery from gambling problems: A comparison of resolved and active gamblers. Addiction, 95, 777-789.

Ibáñez, A. y Saiz, J. (2000). La ludopatía: una "nueva" enfermedad. Barcelona. Masson. 
Irurita, I.M. (1996). Estudio sobre la prevalencia de los jugadores de azar en Andalucía. Sevilla. Comisionado para la Droga, Consejería de Asuntos Sociales, Junta de Andalucía.

Ladouceur, R., Grosselin, P., Laberge, M. y Blaszcynski, A. (2001). Dropouts in clinical research: Do results reflect clinical reality?. Behavior Therapist, 24, 44-46.

Ladouceur, R., Sylvain, C., Boutin, C., Lachance, S., Doucet, C. y Leblond, J. (2003). Group therapy for pathological gamblers: A cognitive approach. Behaviour Research and Therapy, 41, 587-596.

Lesieur, H.R. y Blume, S.B. (1987). The Soath Oaks Gambling Screen (SOGS) : A new instrument for the identification of pathological gamblers. American Journal of Psychiatry, 144, 1184-1188.

Lesieur, H.R. y Heineman, M. (1988). Pathological gambling among youthful multiple substance abusers in a therapeutic community. British Journal of Addictions, 83, 765-771

McCormick, R.A., Russo, A., Ramirez, L. y Taber, J. (1984). Affective disorders among pathological gamblers seeking treatment. American Journal of Psychiatry, 141, 215-218.

McLellan, A.T., Luborsky, L., Woody, G.E. y O'Brien, C.P. (1980). An improved diagnostic evaluation instrument for substance abuse patients: The Addiction Severity Index. Journal of Nervous and Mental Disease, 168, 26-33.

Milton, S. (2001). Stop gambling. A self-help manual for giving up gambling. Sydney, Australia.
Milton, S., Crino, R., Hunt, C. y Proser, E. (2002). The effect of compliance-improving interventions on the cognitivebehavioural treatment of pathological gambling. Journal of Gambling Studies, 18, 207-229.

Nathan, P. (2003). The role of natural recovery in alcoholism and pathological gambling. Journal of Gambling Studies, 19, 279-286.

Petry, N. (2003). Validity of a gambling scale for the Addiction Severity Index. Journal of Nervous and Mental Disease, 191, 399-407.

Prieto, M. (2003). Una propuesta de clasificación de los jugadores patológicos. Psicología Conductual, 11, 523

Prochaska, J.O. y DiClemente, C.C. (1982). Thanstheoretical therapy: Toward a more integrative model of change. Psychotherapy: Theory, Research and Practice, 19, 276-288.

Raylu, N. y Oei, T. (2002). Pathological gambling. A comprehensive review. Clinical Psychology Review, 22, 1009-1061.

Rodríguez-Martos, A. (1989). Estudio piloto estimativo de la prevalencia de juego patológico entre los pacientes alcohólicos que acuden al Programa DROSS. Revista Española de Drogodependencias, 14, 265-275.

Tejeiro, R. (1996). Estudio sobre el juego de azar en Algeciras. Prevalencia y características sociodemográficas. Algeciras. JARCA.

Toneatto, T. y Ladouceur, R. (2003). Treatment of pathological gambling: A critical review of the literature. Psychology of Addictive Behaviors, 17, 284-292. 
